\section{RSP}

http://www.rsp.fsp.usp.br/
Revista de Saúde Pública

\title{
Oferta de serviços pela atenção básica e detecção da incidência de tuberculose no Brasil
}

Daniele Maria Pelissari', Patricia Bartholomay', Marina Gasino Jacobs', Denise Arakaki-Sanchez', Davllyn Santos Oliveira dos Anjos", Mara Lucia dos Santos Costa", Pauline Cristine da Silva Cavalcanti"I, Fredi Alexander Diaz-Quijano ${ }^{\text {IV }}$

Ministério da Saúde. Coordenação Geral do Programa Nacional de Controle de Tuberculose. Brasília, DF, Brasil

" Ministério da Saúde. Coordenação Geral de Acompanhamento e Avaliação da Atenção Básica. Brasília, DF, Brasil

III Fundação Oswaldo Cruz. Centro de Pesquisa Aggeu Magalhães. Programa de Pós-Graduação em Saúde Coletiva. Recife, PE, Brasil

Iv Universidade de São Paulo. Faculdade de Saúde Pública. Departamento de Epidemiologia. São Paulo, SP, Brasil

\section{RESUMO}

OBJETIVO: Avaliar a associação entre os serviços de saúde ofertados por equipes de atenção básica e a detecção de casos novos de tuberculose no Brasil.

MÉTODOS: Estudo ecológico, abrangendo todos os municípios brasileiros que registraram pelo menos um caso novo de tuberculose (diagnosticado entre 2012 a 2014 e notificado no Sistema de Informação de Agravos de Notificação) e com pelo menos uma equipe de atenção básica avaliada pelo segundo ciclo do Programa Nacional de Melhoria do Acesso e da Qualidade da Atenção Básica (PMAQ-AB). As variáveis do PMAQ-AB foram classificadas como próximais ou distais, segundo a sua relação com o diagnóstico de tuberculose. Em seguida, foram testadas hierarquicamente em modelos múltiplos (ajustados por Unidade Federada), usando regressão binomial negativa.

Correspondência:

Daniele Maria Pelissari

SRTVN Quadra 701 Via W 5 Norte Lote D Edifício PO700

70719-040 Brasília, DF, Brasil

E-mail: daniele.pelissari@saude.gov.br

Recebido: 20 mai 2017

Aprovado: 10 jul 2017

Como citar: Pelissari DM, Bartholomay P, Jacobs MG, Arakaki-Sanchez D, Anjos DSO, Costa MLS, et al. Oferta de serviços pela atenção básica e detecção da incidência de tuberculose no Brasil. Rev Saude Publica. 2018;52:53.

Copyright: Este é um artigo de acesso aberto distribuído sob os termos da Licença de Atribuição Creative Commons, que permite uso irrestrito, distribuição e reprodução em qualquer meio, desde que o autor e a fonte originais sejam creditados.
RESULTADOS: Um incremento de 10\% na cobertura da atenção básica esteve associado à redução de 2,24\% na taxa de detecção de tuberculose (IC95\% -3,35- -1,11). No que se refere às variáveis proximais ao diagnóstico, no modelo múltiplo, a detecção da tuberculose esteve associada à proporção de equipes que: realizam vigilância de contatos (incremento na Razão de Taxas de Incidência [RTI] = 2,97\%; IC95\% 2,41-3,53); fazem busca ativa de casos de tuberculose (incremento na RTI = 2,17\%; IC95\% 1,48-2,87); e, ofertam cultura para micobactérias (incremento na RTI = 1,87\%; IC95\% 0,98-2,76).

CONCLUSÕES: As variáveis relacionadas às ações de detecção estiveram positivamente associadas à detecção de casos novos de tuberculose, sugerindo uma contribuição significativa ao fortalecimento da sensibilidade do sistema de vigilância. Por outro lado, a cobertura da atenção básica esteve inversamente associada à taxa de detecção de tuberculose, o que poderia representar o efeito global da atenção básica sobre o controle da transmissão, provavelmente, através da identificação e tratamento precoce de casos.

DESCRITORES: Tuberculose, epidemiologia. Tuberculose, diagnóstico. Atenção Primária à Saúde. Qualidade, Acesso e Avaliação da Assistência à Saúde. 


\section{INTRODUÇÃO}

Foram registrados 66.796 casos novos de tuberculose (TB) no Brasil em 2016, perfazendo um coeficiente de incidência de 32,4/100 mil habitantes (hab.) a. Apesar do diagnóstico da doença ser gratuito no país, segundo a Organização Mundial de Saúde (OMS), 13\% das pessoas com a doença não foram detectadas no Brasil nesse mesmo ano ${ }^{\mathrm{b}}$. Os casos não identificados e, consequentemente, não tratados, constituem reservatórios da doença e, portanto, um desafio para o seu controle ${ }^{1}$.

A cobertura populacional estimada de atenção básica (AB) foi de 69,3\% em 2013․ Nesse mesmo ano, $61,4 \%$ dos casos novos pulmonares bacilíferos foram diagnosticados e tratados por esses serviços nas capitais brasileiras ${ }^{2}$. No entanto, na região Nordeste, $46,5 \%$ dos usuários procuraram os serviços da $\mathrm{AB}$ no início dos sinais e sintomas da TB³ ${ }^{3}$. Em Pelotas, RS, 24,4\% dos sintomáticos respiratórios procuraram a $\mathrm{AB}$, dos quais menos da metade teve acesso aos exames laboratoriais para o diagnóstico de TB devido à não solicitação pelos profissionais de saúde 4 .

Entre os fatores associados à procura desses serviços no país está a proximidade da unidade de $\mathrm{AB}$, o tempo de espera para a consulta, e o vínculo com a equipe de saúde ${ }^{3-5}$. A provisão de insumos e recursos humanos ${ }^{6}$ e a presença de equipes de estratégias de saúde da família (ESF) nas unidades de saúde ${ }^{7}$ estão positivamente associadas à detecção de casos. Na Etiópia, o atraso ao diagnóstico esteve associado ao não conhecimento sobre a TB e à distância do serviço de saúde da residência do paciente ${ }^{8}$. De forma semelhante, uma meta-análise de estudos realizados na China identificou, entre outros fatores, que residir distante dos serviços de saúde (área rural) esteve associado ao atraso na detecção de TB9.

a Ministério da Saúde (BR),

Secretaria de Vigilância em Saúde. Perspectivas brasileiras para o fim da tuberculose como problema de saúde pública. Bol Epidemiol. 2016 [citado $2 \mathrm{dez}$ 2016];47(13):1-15. Disponível em: http://portalarquivos. saude.gov.br/images/ pdf/2016/marco/24/2016-009 Tuberculose-001.pdf

${ }^{\mathrm{b}}$ World Health Organization. Global tuberculosis report 2016. Geneva: WHO; 2016 [citado 22 jan 2017]. Disponível em: http://apps.who. int/medicinedocs/documents/ s23098en/s23098en.pdf

' Ministério da Saúde (BR), DATASUS -Departamento de Informática do Sistema Único de Saúde. Informações de saúde (TABNET): assistência à saúde. 2016 [citado 2 dez 2016]. Disponível em: http://www2. datasus.gov.br/DATASUS/index. php? area $=0202$

d Ministério da Saúde (BR), Secretaria de Atenção à Saúde. Programa Nacional de Melhoria do Acesso e da Qualidade da Atenção Básica (PMAQ). Brasília (DF); 2016 [citado 2 nov 2016]. Disponível em: http://dab.saude. gov.br/portaldab/ape_pmaq.php

e Ministério da Saúde (BR),

Secretaria de Atenção à Saúde, Departamento de Atenção Básica. PMAQ: instrumento de avaliação externa para as equipes de atenção básica. Brasília (DF); 2013 [citado 15 nov 2016]. Disponível em:: http://189.28.128.100/dab/ docs/port aldab/documentos/ instrumentos_ae_sfp.pdf

No Brasil, o Programa Nacional de Melhoria do Acesso e da Qualidade da Atenção Básica (PMAQ-AB) visa avaliar a melhoria do atendimento prestado à população pela $\mathrm{AB}$. Essa avaliação contempla aspectos específicos sobre a TB, em especial sobre a detecção de pessoas com a doença ${ }^{d}$. Estudos de abrangência nacional sobre os fatores associados à detecção de casos de TB podem gerar evidências para o fortalecimento de políticas públicas de saúde. Assim, o PMAQ-AB torna-se uma fonte de dados robusta para esse tipo de estudo, tanto em razão de sua abrangência nacional, quanto por ser uma ferramenta qualificada para avaliar diversas dimensões sobre o cuidado às pessoas com TB.

Nesse contexto, este estudo teve por objetivo analisar a associação entre os serviços de saúde ofertados por equipes de atenção básica (eAB) e a detecção de casos novos de TB no Brasil.

\section{MÉTODOS}

Estudo ecológico sobre a taxa registrada de incidência da TB, também denominada para fins deste estudo como taxa de detecção, e sua associação com os serviços de saúde ofertados por eAB no Brasil entre 2012 e 2014.

Uma vez que a incidência de casos notificados é resultante da presença dos eventos na comunidade (incidência propriamente dita) e da sensibilidade do sistema de vigilância (detecção) $^{10}$, trabalhou-se com a hipótese de que uma melhora na oferta de serviços diagnósticos estaria associada à maior detecção de casos e, em consequência, à maior incidência aparente. Assim, para os fins deste manuscrito, assumiu-se que as diferenças na taxa, associadas às ações das eAB, representam mais contribuições à sensibilidade do sistema do que mudanças no risco de adoecer.

Para medir a oferta de serviços de saúde por eAB, foram utilizados os dados do segundo ciclo do PMAQ-AB do Módulo II do "Instrumento de avaliação externa para as equipes de atenção básica”. Esta avaliação foi aplicada aos profissionais das equipes entre novembro de $2013 \mathrm{e}$ junho de 2014, e coletou dados de 29.778 equipes em 24.038 unidades básicas de saúde de 5.043 municípios ${ }^{\mathrm{d}}$. Nessa avaliação, algumas perguntas específicas sobre TB foram realizadas apenas para equipes que possuíam registro de casos de TB identificados no ano anterior à avaliação. 
A população de estudo foi composta pelos municípios brasileiros que registraram pelo menos um caso novo de TB diagnosticado entre 2012 a 2014 e notificado no Sistema de Informação de Agravos de Notificação (Sinan) segundo ciclo do PMAQ-AB. As estimativas populacionais por município foram obtidas do Instituto Brasileiro de Geografia e Estatísticag.

A variável dependente foi a taxa registrada de incidência de TB de 2012 a 2014, calculada como a relação da soma de casos novos de TB (todas as formas da doença) e da população estimada do município desse mesmo período. Esse período foi definido com base na proximidade do momento da avaliação do PMAQ- $\mathrm{AB}$ e na disponibilidade dos dados sobre a incidência dos casos de TB no momento da análise.

As variáveis independentes do PMAQ-AB para o nível municipal foram analisadas como a proporção (\%) de equipes que responderam realizar determinada atividade no serviço de $A B$ naquele município. As variáveis selecionadas para análise consistiam naquelas que conceitualmente poderiam explicar a incidência ou a detecção de casos de TB dos blocos:

- Apoio institucional e apoio matricial: equipes com apoio matricial da vigilância em saúde;

- Territorialização e população de referência: equipes com definição da área de abrangência; e, com população sem cobertura pela $\mathrm{AB}$ no entorno do território de abrangência da equipe;

- Acolhimento à demanda espontânea: equipes que realizam avaliação de risco e vulnerabilidade no acolhimento dos usuários;

- Organização da agenda: equipes com agenda organizada para acolhimento à demanda espontânea; e com reserva de vagas na agenda ou um horário de fácil acesso ao profissional para que o usuário possa sanar dúvidas pós-consulta ou mostrar como evoluiu sua situação;

- Atenção à saúde: equipes cuja consulta especializada é agendada pela unidade de saúde; e que realizam busca ativa de casos de TB na população geral;

- Exames solicitados pela equipe de AB: baciloscopia para TB; radiografia de tórax para TB; cultura de micobactérias; sorologia para o HIV; e teste rápido para o HIV;

- Atenção à pessoa com tuberculose: equipes que possuem a estimativa anual de casos de TB e sintomáticos respiratórios; cuja primeira amostra de escarro para o diagnóstico de TB é coletada na primeira consulta; que possuem registro do número de usuários com TB identificados no último ano; com ficha de notificação de casos de TB na unidade; $\mathrm{e}$ que realizam a vigilância de contatos intradomiciliares de casos novos de TB;

- Promoção da Saúde: equipes que realizam grupos com enfoque de orientar sobre doenças transmissíveis.

Também foi analisada a estimativa da cobertura populacional da AB no município em 2013․

${ }^{\mathrm{f}}$ Ministério da Saúde (BR),

DATASUS -Departamento de Informática do Sistema Único de Saúde. Informações de saúde (TABNET): epidemiológicas e morbidade. Brasília (DF); 2016 [citado 2 dez 2016]. Disponível em: http://www2.datasus.gov.br/ DATASUS/index.php?area $=0203$

g Ministério da Saúde (BR), DATASUS -Departamento de Informática do Sistema Único de Saúde. Informações de saúde (TABNET): demográficas e socioeconômicas. Brasília (DF); 2016 [citado 2 dez 2016]. Disponível em: http://www2. datasus.gov.br/DATASUS/index. php?area $=0206$
A mediana e o intervalo interquartil (IQ) foram calculados para cada variável independente. Essas variáveis foram classificadas em distais e proximais em relação ao desfecho. As variáveis proximais foram aquelas que estavam mais diretamente relacionadas à detecção das pessoas com TB, como a busca ativa de casos de TB na população geral e a oferta de exames para o diagnóstico da doença. $\mathrm{E}$ as distais, aquelas que representavam a organização do serviço em geral (ex.: possuir registro do número de usuários com TB), cobertura dos serviços no território (ex.: cobertura de $\mathrm{AB}$ ) e oferta de serviços que poderiam estar indiretamente relacionados à detecção de pessoas com TB (ex.: equipes que realizam grupos com enfoque de orientar sobre doenças transmissíveis).

A análise foi realizada com regressão binomial negativa devido à superdispersão, distribuição e características da variável dependente. As variáveis que apresentaram individualmente associações com $\mathrm{p}<0,20$ foram analisadas em uma matriz de correlação. Com vistas a evitar colinearidade entre as variáveis com correlação maior do que 0,40 foram selecionadas 
para o modelo múltiplo as de maior associação com o desfecho ou com melhor suporte conceitual. Esse processo foi realizado separadamente para as variáveis do modelo distal e proximal.

Seguindo uma ordem hierárquica na análise ${ }^{11}$, foi obtido um modelo múltiplo com as variáveis distais (modelo 1). Nesse modelo, foram conservadas aquelas variáveis com $\mathrm{p}<0,05$. O mesmo processo foi realizado para identificar as variáveis proximais associadas ao desfecho (modelo 2). Por fim, essas últimas associações foram ajustadas pelas variáveis distais do primeiro modelo (modelo 3). Esse último modelo foi aplicado em cada uma das cinco macrorregiões do Brasil para avaliar graficamente a variabilidade das associações.

As medidas de associação estimadas nos modelos de regressão foram apresentadas como o incremento percentual na razão de taxas de incidência (incremento na RTI), a cada acréscimo em 10 pontos percentuais da variável independente correspondente. Todos os modelos incluíram Unidade Federada como variável de ajuste, pois se assumiu a existência de variação entre os diferentes contextos. No entanto, os coeficientes de regressão por Unidade Federada não foram apresentados nas tabelas devido à sua extensão. O Stata versão

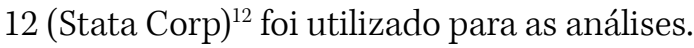

O presente estudo foi aprovado pela Comissão Nacional de Ética em Pesquisa (Parecer 1.810.520 de 12 de novembro de 2016).

\section{RESULTADOS}

Dos 5.043 municípios que possuíam pelo menos uma eAB avaliada pelo PMAQ-AB, 4.428 (79,7\% do total de municípios brasileiros) apresentaram pelo menos um caso novo de TB de 2012 a 2014. Esses municípios incluídos no estudo reportaram 202.092 casos novos de TB nesse mesmo período ( $96,4 \%$ do total de casos incidentes), representando uma média de 67.364 casos por ano.

No que se refere às variáveis distais ao desfecho, a cobertura da $\mathrm{AB}$ foi de $100 \%$ em 63,7\% dos municípios incluídos neste estudo (Percentil 25 da cobertura: 83,1\%). Outras variáveis coletadas no PMAQ-AB também apresentaram alta proporção, como é o caso de equipes que têm a sua área de abrangência definida, que realizam avaliação de risco e vulnerabilidade no acolhimento dos usuários, com apoio matricial da vigilância em saúde, que possuem registro do número de usuários com TB, e que solicitam sorologia para o HIV (Tabela 1).

As seguintes variáveis distais, ajustadas pela Unidade Federada, apresentaram associação significativa com a taxa de detecção de casos de TB: cobertura de AB; proporção de equipes com população descoberta pela AB; proporção de equipes com agenda organizada para acolhimento à demanda espontânea; proporção de equipes cuja consulta especializada é marcada pela unidade de saúde; proporção de equipes que solicitam sorologia para o HIV; e teste rápido para o HIV ou qualquer um dos dois testes (Tabela 1).

A variável "proporção de equipes que ofertam sorologia ou teste rápido para o HIV" esteve fortemente correlacionada com a "oferta de sorologia para o HIV" (Spearman 0,65). Optou-se por incluir esta última no modelo 1 por estar mais fortemente associada à detecção de casos de TB.

Quanto às variáveis proximais, a oferta de exames de radiografia de tórax e baciloscopia para a TB apresentaram elevada cobertura entre as equipes. No entanto, atividades como a busca ativa de casos de TB na população geral (mediana $=70,6$; IQ = 40-100) e a coleta da primeira amostra de escarro para o diagnóstico de TB na primeira consulta (mediana $=50$; $\mathrm{IQ}=22,2-94,4)$ não se mostraram como atividades plenamente incorporadas pelas equipes nos municípios (Tabela 2). 
Tabela 1. Análise descritiva e associação entre variáveis distais e a taxa de detecção da tuberculosea . Brasil, 2012 a 2014.

\begin{tabular}{|c|c|c|}
\hline Variáveis distais (a cada 10\%) & Mediana (IQ) & $\begin{array}{l}\text { Incremento na RTI } \\
\quad(\text { IC95\%) }\end{array}$ \\
\hline \% de cobertura de atenção básica & $100(83,1-100)$ & $-1,88(-2,87--0,88)^{\mathrm{d}}$ \\
\hline \% de equipes com definição da área de abrangência & $100(100-100)$ & $0,21(-1,75-2,21)$ \\
\hline \% de equipes com população descoberta pela atenção básica no entorno do território de abrangência & $10(0-50)$ & $2,23(1,58-2,88)^{\mathrm{d}}$ \\
\hline \% de equipes que realizam avaliação de risco e vulnerabilidade no acolhimento dos usuários & $100(87,5-100)$ & $-0,65(-1,71-0,41)$ \\
\hline \% de equipes com agenda organizada para acolhimento à demanda espontânea & $100(66,7-100)$ & $1,42(0,6-2,25)^{\mathrm{d}}$ \\
\hline $\begin{array}{l}\text { \% de equipes com reserva de vagas na agenda ou um horário de fácil acesso ao profissional para } \\
\text { que o usuário possa sanar dúvidas pós-consulta ou mostrar como evoluiu sua situação }\end{array}$ & $75(50-100)$ & $0(-0,7-0,69)$ \\
\hline \% de equipes cuja consulta especializada é marcada pela unidade de saúde & $40(0-90,9)$ & $1,64(1,05-2,23)^{\mathrm{d}}$ \\
\hline $\begin{array}{l}\text { \% de equipes que realizam grupos com enfoque de orientar sobre doenças transmissíveis (dengue, } \\
\text { tuberculose, hanseníase, HIV, tracoma) }\end{array}$ & $75(50-100)$ & $0,52(-0,14-1,19)$ \\
\hline \% de equipes com apoio matricial da vigilância em saúde & $100(80-100)$ & $-0,1(-0,92-0,74)$ \\
\hline $\begin{array}{l}\text { \% de equipes que possuem registro do número de usuários com tuberculose, identificados no } \\
\text { último ano }\end{array}$ & $100(100-100)$ & $0,72(-0,88-2,35)$ \\
\hline$\%$ de equipes que solicitam sorologia $\mathrm{HIV}^{\mathrm{c}}$ & $100(100-100)$ & $2,8(1,24-4,4)^{\mathrm{d}}$ \\
\hline$\%$ de equipes que solicitam teste rápido HIV ${ }^{c}$ & $87,1(33,3-100)$ & $0,75(0,11-1,38)^{\mathrm{d}}$ \\
\hline$\%$ de equipes que solicitam teste rápido ou sorologia para o HIV c & $100(100-100)$ & $2,44(0,02-4,93)^{d}$ \\
\hline
\end{tabular}

IQ: intervalo interquartil; RTI: razão de taxas de incidência

a A medida de associação representa o incremento na razão de taxas de incidência (RTI-1) expressada em percentagem a cada $10 \%$ da variável independente junto com o intervalo com 95\% de confiança (IC95\%). Todas as medidas de associação estão ajustadas por Unidade Federada.

${ }^{\mathrm{b}}$ Com documento que comprove.

c São realizados pela rede de serviços de saúde.

${ }^{\mathrm{d}} \mathrm{p}<0,05$

Tabela 2. Análise descritiva e associação das variáveis proximais com a taxa de detecção da tuberculose . Brasil, 2012 a 2014.

\begin{tabular}{|c|c|c|}
\hline Variáveis proximais (a cada $10 \%$ ) & Mediana (IQ) & $\begin{array}{l}\text { Incremento na RTI } \\
\quad(\text { IC95\%) }\end{array}$ \\
\hline$\%$ de equipes que realizam busca ativa para TB & $70,6(40-100)$ & $3,15(2,46-3,84)^{\mathrm{d}}$ \\
\hline$\%$ de equipes que solicitam raio- $X \mathrm{~TB}^{\mathrm{b}}$ & $100(100-100)$ & $2,43(1,18-3,69)^{\mathrm{d}}$ \\
\hline$\%$ de equipes que solicitam baciloscopia para $\mathrm{TB}^{\mathrm{b}}$ & $100(100-100)$ & $3,8(2,26-5,36)^{\mathrm{d}}$ \\
\hline$\%$ de equipes que solicitam cultura de micobactérias ${ }^{b}$ & $100(71,4-100)$ & $2,36(1,46-3,27)^{\mathrm{d}}$ \\
\hline $\begin{array}{l}\% \text { de equipes que possuem a estimativa anual de número de casos de TB e sintomáticos } \\
\text { respiratórios em seu território }\end{array}$ & $93,8(66,7-100)$ & $2,19(1,4-2,99)^{\mathrm{d}}$ \\
\hline$\%$ de equipes cuja $1^{\mathrm{a}}$ amostra de escarro para o diagnóstico de TB é coletada na primeira consulta & $50(22,2-94,4)$ & $0,81(0,15-1,46)^{\mathrm{d}}$ \\
\hline$\%$ de equipes com ficha de notificação de casos de TB na unidade ${ }^{c}$ & $100(100-100)$ & $0,8(-0,62-2,23)$ \\
\hline \% de equipes que realizam a vigilância de contatos intradomiciliares de casos novos de TB & $100(0-100)$ & $3,56(3,02-4,11)^{\mathrm{d}}$ \\
\hline
\end{tabular}

IQ: intervalo interquartil; RTI: razão de taxas de incidência; TB: tuberculose

a A medida de associação representa o incremento na razão de taxas de incidência (RTI-1) expressada em percentagem a cada $10 \%$ da variável independente junto com o intervalo com 95\% de confiança (IC95\%). Todas as medidas de associação estão ajustadas por Unidade Federada.

${ }^{\text {b }}$ São realizados pela rede de serviços de saúde.

${ }^{c}$ Com documento que comprove.

${ }^{\mathrm{d}} \mathrm{p}<0,05$

Com exceção da "proporção de equipes com ficha de notificação de casos de TB na unidade", as variáveis proximais estiveram significativamente associadas ao desfecho (Tabela 2). A disponibilidade de radiografia de tórax para TB não foi considerada para a análise múltipla, pois apresentou uma importante correlação com a oferta de baciloscopia $($ Spearman $=0,46)$ e, esta última, teve maior associação com a incidência registrada.

No modelo 1, as variáveis distais positivamente associadas à detecção foram as seguintes proporções de equipes: com população sem cobertura pela $\mathrm{AB}$ no entorno do território de abrangência; cuja consulta especializada é marcada pela unidade de saúde; que solicitam sorologia para o HIV; e com agenda organizada para acolhimento à demanda espontânea (Tabela 3). Um aumento de 10,0\% na proporção de cobertura da $\mathrm{AB}$ esteve associado à 
redução de 1,9\% na RTI (IC95\% -3,06- -0,76). Adicionalmente, um aumento de 10,0\% nas equipes que realizam a sorologia para o HIV representou um aumento de $2,4 \%$ no desfecho em estudo (IC95\% 0,81-3,94).

Em relação às variáveis proximais (modelo 2), a taxa de detecção de TB esteve positivamente associada às proporções de equipes que: realizam a vigilância de contatos intradomiciliares; realizam busca ativa de casos de TB na população geral; solicitam baciloscopia para TB; e solicitam cultura de micobactérias (Tabela 3). A vigilância de contatos intradomiciliares e a busca ativa estiveram associadas a um aumento de 3,0\% (IC95\% 2,49-3,6) e 2,1\% (IC95\% 1,37-2,77) na RTI, respectivamente. O aumento desses indicadores representou um acréscimo consistente na taxa de detecção da TB (Figura 1).

Quanto ao modelo final (modelo 3), ajustado por Unidade Federada e variáveis distais, com exceção da proporção de equipes que solicitam baciloscopia para TB, todas as demais variáveis proximais permaneceram significativas (Tabela 3). A Figura 2 apresenta uma descrição das associações do modelo 3, quando aplicado a cada uma das macrorregiões do Brasil.

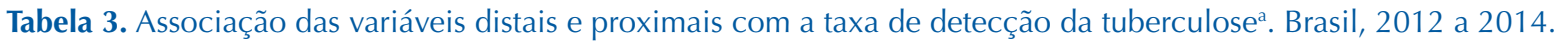

\begin{tabular}{|c|c|c|c|}
\hline Variável (a cada 10\%) & $\begin{array}{c}\text { Modelo } 1 \\
\text { Incremento na RTI } \\
\text { (IC95\%) ajustado }\end{array}$ & $\begin{array}{c}\text { Modelo } 2 \\
\text { Incremento na RTI } \\
\text { (IC95\%) ajustado }\end{array}$ & $\begin{array}{c}\text { Modelo } 3 \\
\text { Incremento na RTI } \\
\text { (IC95\%) ajustado }\end{array}$ \\
\hline $\begin{array}{l}\text { \% de equipes com população descoberta pela atenção básica no } \\
\text { entorno do território de abrangência }\end{array}$ & $1,87(1,2-2,54)$ & & $1,69(1,03-2,34)$ \\
\hline $\begin{array}{l}\text { \% de equipes cuja consulta especializada é marcada pela unidade de } \\
\text { saúde e a data posteriormente informada ao usuário }\end{array}$ & $1,53(0,95-2,12)$ & & $1,33(0,76-1,91)$ \\
\hline \% de cobertura de atenção primária & $-1,92(-3,06--0,76)$ & & $-2,24(-3,35--1,11)$ \\
\hline$\%$ de equipes que solicitam sorologia $\mathrm{HIV}^{\mathrm{b}}$ & $2,37(0,81-3,94)$ & & \\
\hline $\begin{array}{l}\% \text { de equipes com agenda organizada para acolhimento } \\
\text { à demanda espontânea }\end{array}$ & $1,32(0,51-2,14)$ & & \\
\hline $\begin{array}{l}\% \text { de equipes que realizam a vigilância de contatos } \\
\text { intradomiciliares de casos novos de TB }\end{array}$ & & $3,04(2,49-3,6)$ & $2,97(2,41-3,53)$ \\
\hline$\%$ de equipes que realizam busca ativa para TB & & $2,07(1,37-2,77)$ & $2,17(1,48-2,87)$ \\
\hline$\%$ de equipes que solicitam baciloscopia para $\mathrm{TB}^{\mathrm{b}}$ & & $1,91(0,33-3,52)$ & \\
\hline$\%$ de equipes que solicitam cultura de micobactérias ${ }^{b}$ & & $1,61(0,67-2,55)$ & $1,87(0,98-2,76)$ \\
\hline 2Log likelihood & $-28400,7$ & $-28280,0$ & $-28207,6$ \\
\hline
\end{tabular}

RTI: razão de taxas de incidência; TB: tuberculose

2Log likelihood modelo vazio $=-31.217,83$

${ }^{a}$ A medida de associação representa o incremento na razão de taxas de incidência (RTI-1) expressada em percentagem a cada $10 \%$ da variável independente junto com o intervalo com 95\% de confiança (IC95\%). Todas as medidas de associação estão ajustadas por Unidade Federada e pelas demais variáveis incluídas no modelo correspondente. Todas as associações foram estatisticamente significativas.

b São realizados pela rede de serviços de saúde.

(A)

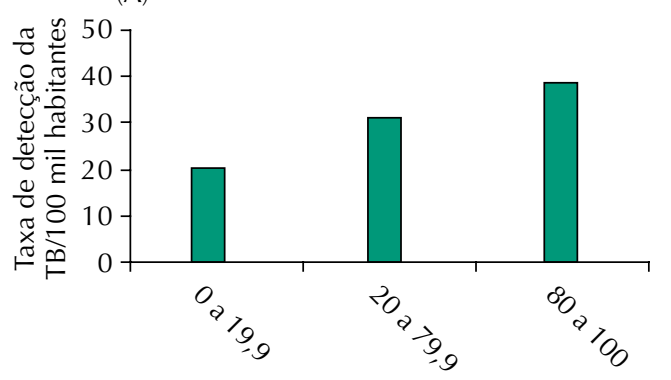

$\%$ de equipes que realizam vigilância de contatos intradomiciliares para casos novos de TB
(B)

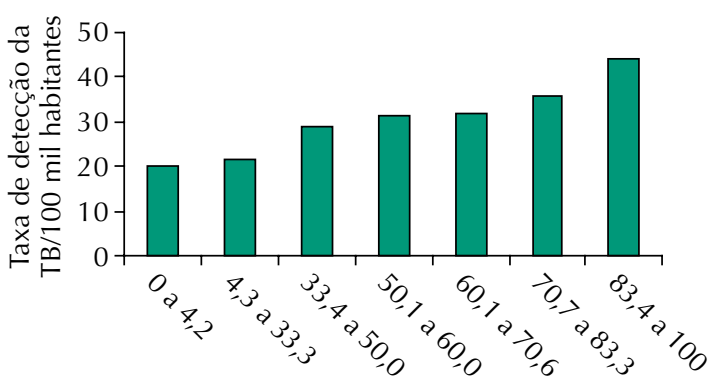

$\%$ de equipes que realizam busca ativa de casos de TB na população geral

Figura 1. Taxa de detecção de tuberculose (TB) segundo a proporção de equipes que realizam vigilância de contatos e busca ativa para tuberculose. Brasil, 2012 a 2014. (A) Vigilância de contatos intradomiciliares de casos novos de TB. (B) Busca ativa de casos de TB na população geral. 


\section{Variáveis Distais}

A) População descoberta pela atenção básica no entorno do território

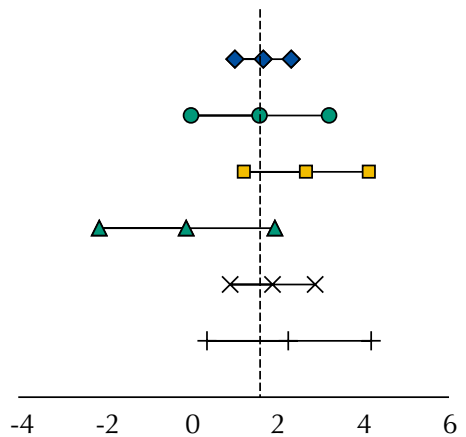

B) Consulta especializada é marcada pela unidade de saúde

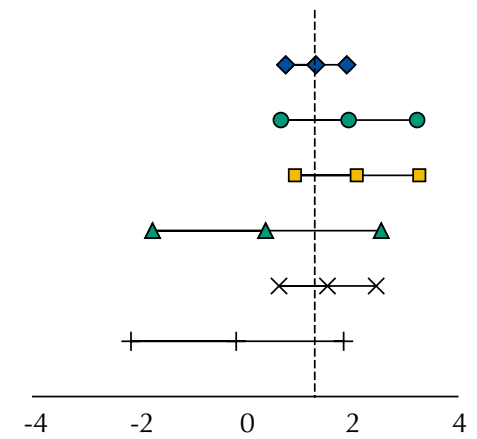

C) Cobertura de atenção primária

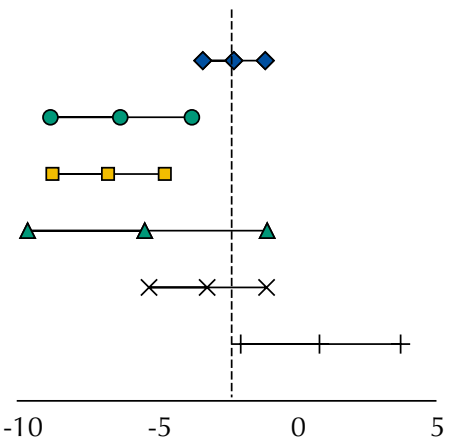

Incremento na razão de taxas de incidência

Variáveis Proximais

E) Realizam busca ativa para TB

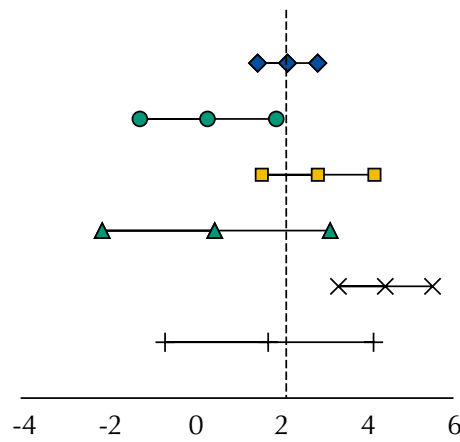

$\multimap$ Brasil

-o- Sul

$\rightarrow-$ Sudeste

$\Delta$ Centro-Oeste

$\times$ Nordeste

+ Norte
D) Realizam a vigilância de contatos intradomiciliares de casos novos de TB

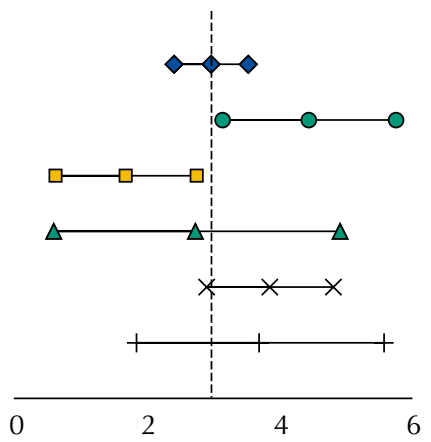

Incremento na razão de taxas de incidência

F) Solicitam cultura de micobactérias

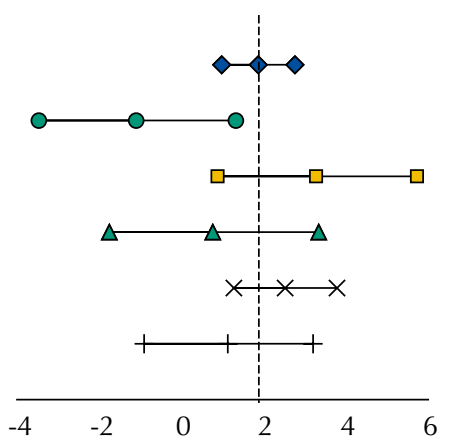

$\checkmark$ Brasil

$\rightarrow-$ Sul

$\neg-$ Sudeste

$\neg-$ Centro-Oeste

$\leftarrow$ Nordeste

+ Norte

Os gráficos representam a associação de cada uma das variáveis do modelo 3, ajustada pelas demais quando aplicada aos dados de cada uma das regiões. (A) População descoberta pela atenção básica no entorno do território. (B) Consulta especializada é marcada pela unidade de saúde. (C) Cobertura de atenção primária. (D) Realizam a vigilância de contatos intradomiciliares de casos novos de TB. (E) Realizam busca ativa para TB na população geral. (F) Solicitam cultura de micobactérias e são realizados pela rede de serviços de saúde.

Figura 2. Variáveis associadas à taxa de detecção de tuberculose (TB) segundo Macrorregiões. Brasil, 2012 a 2014.

\section{DISCUSSÃO}

Este estudo ecológico, que incluiu aproximadamente $80 \%$ dos municípios brasileiros, identificou a importância de ações realizadas pelas eAB e da cobertura populacional estimada da $A B$ na detecção de casos incidentes de TB. A maioria das variáveis coletadas pelo PMAQ-AB está associada positivamente à detecção de casos, sugerindo que as atividades das eAB aumentam a sensibilidade dos serviços. Os resultados destacam a importância da estratégia do modelo de $\mathrm{AB}$ adotado para a detecção de casos de $\mathrm{TB}$ e, consequentemente, para a identificação da endemia oculta no Brasil.

No modelo distal, a proporção de equipes com população descoberta pela $\mathrm{AB}$ no entorno do território esteve positivamente associada à incidência registrada. Em complemento, a cobertura da $\mathrm{AB}$ apresentou associação negativa com o desfecho em estudo. Essas associações sugerem que a cobertura da $A B$ incide sobre $o$ atendimento e o tratamento das pessoas com $\mathrm{TB}$, reduzindo o risco de transmissão da doença. Isso afetaria o desfecho analisado neste estudo, mais sobre o ponto de vista de risco do que sobre a detecção.

Por outro lado, variáveis como: a marcação de consultas especializadas pela equipe, a oferta de sorologia para o HIV, e a organização de agenda para demanda espontânea, estiveram associadas ao aumento da taxa de detecção. Essas ações, embora não diretamente relacionadas à TB, 
indicam que a oferta de ações pelas equipes de $\mathrm{AB}$ e a organização desses serviços podem favorecer o diagnóstico da doença e levar a um aumento da sensibilidade do sistema de vigilância.

Quanto ao modelo final, cujas variáveis proximais foram ajustadas pelas distais, ações realizadas pelas eAB, diretamente implicadas na detecção de casos de TB, estiveram positivamente associadas ao registro de casos incidentes. É o caso da vigilância de contatos, a busca ativa de casos de TB na população geral, e a oferta de cultura para micobactérias. Esses resultados apontam a importância dessas ações como estratégias que devem ser fortalecidas para aumentar a detecção de casos.

As variáveis analisadas neste estudo não foram diretamente avaliadas em estudos prévios, ou pelo menos não no nível ecológico ${ }^{7,13,14}$. No entanto, indiretamente, um estudo realizado no Sul do país, constatou que unidades de saúde com ESF apresentaram melhores resultados para deteç̧ão de casos de TB quando comparadas com unidades sem essa estratégia 7 . A ESF possui características assistenciais que ultrapassam as barreiras físicas das unidades de saúde ${ }^{13}$, em função da atuação de equipes multidisciplinares e da cobertura dos agentes comunitários de saúde do territóriio ${ }^{\mathrm{h}}$. As ESF estão na $\mathrm{AB}$, que dada a sua relação com a população no território, encontram-se na melhor posição para promover atividades de intervenção de educação em saúde, identificação de grupos de risco e a busca de sintomáticos respiratórios ${ }^{14}$.

A adequada provisão de insumos e recursos humanos, bem como a solicitação de baciloscopia de escarro foram associadas positivamente à detecção de casos em serviços de $\mathrm{AB}$, quando comparados com serviços de Pronto Atendimento em Ribeirão Preto, $\mathrm{SP}^{6}$.

Estudo conduzido na África mostrou que a investigação de contatos intradomiciliares foi mais custo efetiva do que a busca ativa de casos de TB na população geral ${ }^{15}$. No entanto, em Camboja, na Ásia, a busca ativa foi mais custo efetiva em comparação à busca passiva de $\operatorname{casos}^{16}$. No atual estudo, essas duas estratégias foram fatores independentemente associados à taxa de detecção sugerindo que poderiam se complementar para aumentar a sensibilidade do sistema de vigilância.

Quanto aos resultados obtidos no modelo 3 por macrorregiões, com algumas exceções, a maioria dos intervalos de confiança dos estimados regionais incluiu o estimado para o Brasil. Entre as exceções, destaca-se o percentual de cobertura da $A B$, cuja associação negativa foi mais evidente nas regiões Sul e Sudeste. Uma das possíveis explicações para essa variabilidade é a de que os sistemas de informação e a sensibilidade da vigilância sejam melhores nesses locais. Isso facilitaria a medição do efeito benéfico da cobertura da AB sobre a incidência de TB.

No entanto, as variações observadas entre as regiões poderiam ser consideradas aleatórias por permanecerem compatíveis com as associações nacionais, uma vez que nenhuma associação no nível regional atingiu uma significância estatística em direção inversa ao estimado global. Portanto, o modelo final seria suficientemente robusto para suportar as conclusões para o nível nacional.

Entre as limitações deste estudo, está a impossibilidade de estabelecer uma ordem cronológica sobre as variáveis independentes e o desfecho ${ }^{8}$. A organização dos serviços de $A B$ pode ser uma resposta perante uma elevada incidência da doença. De outra forma, espera-se que serviços organizados sejam mais efetivos na detecção de casos incidentes. Além disso, as medidas agregadas usadas em estudos ecológicos podem diferir das individuais ${ }^{17}$. Pelo anterior, não é possível estabelecer um vínculo entre as ações específicas na $\mathrm{AB}$ e cada um dos casos novos de $\mathrm{TB}$.

Múltiplos fatores poderiam afetar as associações encontradas, incluindo as variáveis socioeconômicas, que são determinantes da incidência da $\mathrm{TB}^{18,19} \mathrm{e}$ da organização dos serviços de

${ }^{\text {h} M i n i s t e ́ r i o ~ d a ~ S a u ́ d e ~(B R), ~}$ Secretaria de Atenção à Saúde, Departamento de Atenção Básica. Política nacional de atenção básica. Brasília (DF); 2017 [citado 31 jan 2018]. (Série E. Legislação em Saúde). Disponível em: http://dab.saude. gov.br/portaldab/pnab.php saúde ${ }^{20-22}$. No presente estudo, consideramos que essa variabilidade foi parcialmente controlada com o ajuste da Unidade Federada nos modelos. Além disso, os modelos permaneceram relativamente estáveis pelas regiões, o que sugere que as associações encontradas podem ser relativamente estáveis e aplicáveis a diferentes cenários.

A seleção das equipes avaliadas pelo PMAQ-AB ocorre por adesão e não por amostragem probabilística. As medidas de associação podem ter sido afetadas, caso a adesão ao Programa 
esteja associada à qualidade dos serviços ofertados pelas equipes. No entanto, do total de equipes que cumpriam os requisitos para a avaliação no segundo ciclo ${ }^{\mathrm{d}}, 88,7 \%$ foram avaliadas ${ }^{\mathrm{i}}$.

Apesar dessas limitações, nosso modelo teve por base dados de abrangência nacional, de um sistema de informação que agrega todas as notificações de TB do país e da avaliação realizada a partir do PMAQ-AB, o único programa de avaliação da $\mathrm{AB}$ com envergadura tão ampla no país. Nossa amostra de 4.428 municípios permitiu a avaliação simultânea de diferentes indicadores do PMAQ-AB. Além disso, a elevada variabilidade de nossas unidades de análise permite a extrapolação dos resultados para outros locais. Em contrapartida, as associações de estratégias específicas e sua relação com a detecção de casos é consistente e coerente com o esperado. Dessa forma, este estudo ecológico fornece um panorama que pode orientar a tomada de decisões em políticas públicas, em particular, aquelas relativas ao fortalecimento do sistema de vigilância em saúde pública.

Em 2015, a OMS estimou que foram detectados $87 \%$ dos casos novos de TB no Brasil , e em 2013, ano de realização deste estudo, essa estimativa foi de $82 \%$. A associação positiva entre as ações ofertadas pelas eAB e a identificação de casos de TB, salienta a necessidade de manter um sistema de vigilância ativo para a detecção da endemia oculta da doença no país.

Desde 2004, o Programa Nacional de Controle da Tuberculose (PNCT) recomenda que a detecção e o acompanhamento das pessoas com $\mathrm{TB}$ sejam realizados em serviços de $\mathrm{AB}^{23}$. Mais esforços devem ser implementados para a ampliação da $\mathrm{AB}$ no país, bem como para a incorporação das atividades do controle da TB pelas eAB existentes. A falta de adesão às ações para o controle pode ser fortemente determinada por limitações institucionais, como documentado em diferentes serviços ${ }^{24}$. No âmbito do atendimento básico, essas barreiras podem incluir a alta rotatividade dos profissionais de saúde, dificuldades em atrair médicos qualificados para trabalhar em áreas remotas, e a dificuldade em absorver todas as demandas do território ${ }^{13}$.

Este estudo, que analisa dados do PMAQ-AB e sua relação com a detecção de TB, mostrou que esse instrumento de avaliação possui potencialidades estratégicas a serem exploradas pelos diferentes níveis de gestão do Sistema Único de Saúde (SUS). Apesar das limitações pontuadas, estudos futuros devem considerá-lo como uma importante fonte de dados sobre o retrato da oferta de ações pelas equipes de $\mathrm{AB}$ para o controle da $\mathrm{TB}$ e outras doenças.

Em conclusão, a maioria das ações avaliadas no PMAQ-AB e analisadas neste estudo está associada ao aumento da sensibilidade na detecção de TB nos serviços de AB. No entanto, a cobertura populacional estimada da $\mathrm{AB}$ esteve inversamente associada à taxa de detecção de TB. Essa associação poderia representar o efeito global da $\mathrm{AB}$ sobre o controle da transmissão, provavelmente pela detecção e tratamento precoce de casos.

\section{REFERÊNCIAS}

1. Esmail H, Barry CE 3rd, Young DB, Wilkinson RJ. The ongoing challenge of latent tuberculosis. Philos Trans R Soc Lond B Biol Sci. 2014;369(1645):20130437. https://doi.org/10.1098/rstb.2013.0437

2. Bartholomay P, Oliveira GP, Pinheiro RS, Vasconcelos AMN. Melhoria da qualidade das informações sobre tuberculose a partir do relacionamento entre bases de dados. Cad Saude Publica. 2014;30(11):2459-70. https://doi.org/10.1590/0102-311X00116313

3. Paiva RCG, Nogueira JA, Sá LD, Nóbrega RG, Trigueiro DRSG, Villa TCS. Acessibilidade ao diagnóstico de tuberculose em município do Nordeste do Brasil: desafio da atenção básica. Rev Eletron Enferm. 2014;16(3):520-6. https://doi.org/10.5216/ree.v16i3.23491

iCavalcanti PCS. Atenção básica no Brasil: desafios e iniciativas para qualificação [dissertação]. Brasília (DF): Ủniversidade de Brasília; 2014.

iWorld Health Organization. Global tuberculosis report 2014. Geneva: WHO; 2014 [citado 22 jan 2017]. Disponível em: apps.who.int/ iris/ bitstream/10665/137094/1/ 9789241564809_eng.pdf
4. Antunes LB, Tomberg JO, Harter J, Lima LM, Beduhn DAV, Gonzales RIC. Sintomático respiratório de tuberculose na atenção primária: avaliação das ações segundo as recomendações nacionais. Rev RENE. 2016;17(3):409-15. https://doi.org/10.15253/2175-6783.2016000300015

5. Neves RR, Ferro PS, Nogueira LMV, Rodrigues ILA. Acesso e vínculo ao tratamento de tuberculose na atenção primária em saúde. Rev Pesq Cuid Fundam. 2016;8(4):5143-9. https://doi.org/10.9789/2175-5361.2016.v8i4.5143-5149

6. Andrade RLP, Scatolin BE, Wysocki AD, Beraldo AA, Monroe AA, Scatena LM, et al. Diagnóstico da tuberculose: atenção básica ou pronto atendimento? Rev Saude Publica. 2013;47(6):1149-58. https://doi.org/10.1590/S0034-8910.2013047004650 
7. Cardozo-Gonzales RI, Palha PF, Harter J, Alarcon E, Lima LM, Tomberg JO. Avaliação das ações de detecção de casos de tuberculose na atenção primária. Rev Eletron Enferm. 2015;17(4):1-8. https://doi.org/10.5216/ree.v17i4.32846

8. Yirgu R, Lemessa F, Hirpa S, Alemayehu A, Klinkenberg E. Determinants of delayed care seeking for TB suggestive symptoms in Seru district, Oromiya region, Ethiopia: a community based unmatched case-control study. BMC Infect Dis. 2017;17(1):292. https://doi.org/10.1186/s12879-017-2407-8

9. Li Y, Ehiri J, Tang S, Li D, Bian Y, Lin H, et al. Factors associated with patient, and diagnostic delays in Chinese TB patients: a systematic review and meta-analysis. BMC Med. 2013;11:156. https://doi.org/10.1186/1741-7015-11-156

10. Rothman KJ, Greenland S, Lash TL. Modern epidemiology. 3.ed. Philadelphia: Lippincott Williams \& Wilkins; 2008.

11. Victora CG, Huttly SR, Fuchs SC, Olinto MT. The role of conceptual frameworks in epidemiological analysis: a hierarchical approach. Int J Epidemiol. 1997;26(1):224-7. https://doi.org/10.1093/ije/26.1.224

12. StataCorp. Stata Statistical Software: release 12. College Station: StataCorp LP; 2011.

13. Victora CG, Barreto ML, Leal MC, Monteiro CA, Schmidt MI, Paim JS, et al. Health conditions and health-policy innovations in Brazil: the way forward. The Lancet. 2011 maio:90-102. https://doi.org/10.1016/S0140-6736(11)60055-X

14. Sulis G, Centis R, Sotgiu G, D'Ambrosio L, Pontali E, Spanevello A, et al. Recent developments in the diagnosis and management of tuberculosis. NPJ Prim Care Respir Med. 2016;26:16078. https://doi.org/10.1038/npjpcrm.2016.78

15. Sekandi JN, Dobbin K, Oloya J, Okwera A, Whalen CC, Corso PS. Cost-effectiveness analysis of community active case finding and household contact investigation for tuberculosis case detection in urban Africa. PloS One. 2015;10(2):e0117009. https://doi.org/10.1371/journal.pone.0117009

16. Eang MT, Satha P, Yadav RP, Morishita F, Nishikiori N, Van- Maaren P, et al. Early detection of tuberculosis through community-based active case finding in Cambodia. BMC Public Health. 2012;12:469. https://doi.org/10.1186/1471-2458-12-469

17. Schwartz S. The fallacy of the ecological fallacy: the potential misuse of a concept and the consequences. Am J Public Health. 1994 [citado 2 dez 2016];84(5):819-24. Disponível em: https://www.ncbi.nlm.nih.gov/pmc/articles/PMC1615039/pdf/amjph00456-0117.pdf

18. Pelissari DM, Diaz-Quijano FA. Household crowding as a potential mediator of socioeconomic determinants of tuberculosis incidence in Brazil. PLoS One. 2017;12(4):e0176116. https://doi.org/10.1371/journal.pone.0176116

19. San Pedro A, Oliveira RM. Tuberculose e indicadores socioeconômicos: revisão sistemática da literatura. Rev Panam Salud Publica. 2013;33(4):294-301. https://doi.org/10.1590/S1020-49892013000400009

20. Scatena LM, Villa TCS, Ruffino-Netto A, Kritski AL, Figueiredo TMRM, Vendramini SHF, et al. Dificuldades de acesso a serviços de saúde para diagnóstico de tuberculose em municípios do Brasil. Rev Saude Publica. 2009;43(3):389-97. https://doi.org/10.1590/S0034-89102009005000022

21. Brito EWG, Silva AKF, Teixeira GGA, Dias GBS, Costa NDL, Uchôa SAC. Organização do cuidado à tuberculose na atenção básica do Rio Grande do Norte. Rev Enferm UFPE 2015;9(6 Supl):8643-52. https://doi.org/10.5205/reuol.7061-61015-5-SM0906supl201503

22. Gonzales RIC, Monroe AA, Assis EG, Palha PF, Villa TCS, Ruffino-Netto A, et al. Desempenho de serviços de saúde no tratamento diretamente observado no domicílio para controle da tuberculose. Rev ESC Enferm USP. 2008;42(4):628-34. https://doi.org/10.1590/S0080-62342008000400003

23. Figueiredo TMRM, Villa TCS, Scatena LM, Gonzales RIC, Ruffino-Netto A, Nogueira JA, et al. Desempenho da atenção básica no controle da tuberculose. Rev Saude Publica. 2009;43(5):825-31. https://doi.org/10.1590/S0034-89102009005000054

24. Chapman HJ, Veras-Estévez BA, Pomeranz JL, Pérez-Then EN, Marcelino B, Lauzardo M. Perceived barriers to adherence to tuberculosis infection control measures among health care workers in the Dominican Republic. MEDICC Rev. 2017;19(1):16-22. https://doi.org/10.1590/medicc.2017.190100006

Contribuição dos Autores: DMP, FADQ, MGJ e PB conceberam e planejaram o estudo. DMP e FADQ realizaram as ilustrações. DMP fez a redação da primeira versão. Todos os autores analisaram e interpretaram os dados, realizaram revisão crítica relevante do conteúdo intelectual, aprovaram a versão final e são responsáveis por todos os aspectos do trabalho na garantia da exatidão e integridade de qualquer parte da obra.

Conflito de Interesses: Os autores declaram não haver conflito de interesses. 